\title{
Database Design on Construction Project Cost System
}

\author{
Nannan Zhang ${ }^{1, a}$, Wenfeng Song ${ }^{2, b}$ \\ ${ }^{1}$ School of Management, Bohai University, Jinzhou, 121013, China \\ ${ }^{2}$ State Grid Dalian Electric Power Supply Company, Dalian, 116001, China \\ a331263907@qq.com, b13909863238@139.com
}

\begin{abstract}
Keywords: construction project; cost system; database design; conceptual structure; logical structure; storage structure; index design
\end{abstract}

\begin{abstract}
Construction project cost management is an important part of the construction project management, for the problem existed in the traditional construction project cost management developing construction project cost system to improve the level of management and information. Based on the DB2 database management system database design, provide a viable solution for system development. Design mainly includes three aspects: Firstly, using the entities association diagram carry out the conceptual structure design; then, based on the DB2 data type, using the UML class diagram carry out the logical structure design; finally, physical structure design, including the storage structure design and index design. This paper fully integrated the characteristics of DB2 database management system; to accelerate the construction project cost system development has an important role.
\end{abstract}

\section{Introduction}

Project cost budget is basic control foundation for the construction project cost budget. For construction project investment enterprises, the use of construction project cost system that can fully tap the business data, in-depth analysis the various factors that reduce the cost of investment projects, to provide decision support for the project investment cost control plan. Through the construction project cost system, can also budget for investment projects, to facilitate enterprises before the investment or tender, to determine investment tender plans and reduce systemic risk. On the other hand, in a market economy environment, construction project enterprises are facing severe competition in the market, the enterprises combined with the actual situation, control costs for investment risk, improve market competitiveness. From the current development trend of the construction project enterprises, the achievement of information technology is an important driving force to promote enterprise development, is also a key factor in improving enterprise competitiveness. Therefore, the development construction project cost system for construction enterprises has a great significance. Database design is an important work for system development, the results of this study provides technical support for system development.

\section{Conceptual Structure Design}

The goal of conceptual structure design is overall database conceptual structure to reflect the organization's information needs, according to a specific method abstracted a data model that does not depend on any particular machine, namely conceptual model. Conceptual structure design is to model for the world of information, design requirements as follows: truly and fully reflect the real world, including links between things and things, to meet the user's data processing requirements; easy to understand, and can use the conceptual model exchange views with users are not familiar with computer, the active participant of user is the key to success for database design; easy to modify, when the application environment and application requirements changed, easy to modify and extend the conceptual model; easy to transform relations, net, and levels data model. Conceptual structure is a common basis of various data models, more machine-independent than data models, more abstract, and thus more stable. Powerful tools to describe the conceptual model is the "Entity - Relationship model (E-R)". Common conceptual model is E-R model, conceptual 
design results of the system is shown in Fig. 1 (omitted the attributes of entities and associated) [1, 2]. In Fig. 1, contains four entities, namely "Project information, Project costs detail, Costs name, Costs category", there are three "one-to-many" links between entities, namely "Project information" entities and "Project costs detail" entity exists a "one-to-many" links, "Costs name" entities and "Project costs detail" entity exists a "one-to-many" links, "Costs category" entities and "Costs name" entity exists a "one-to-many" link.

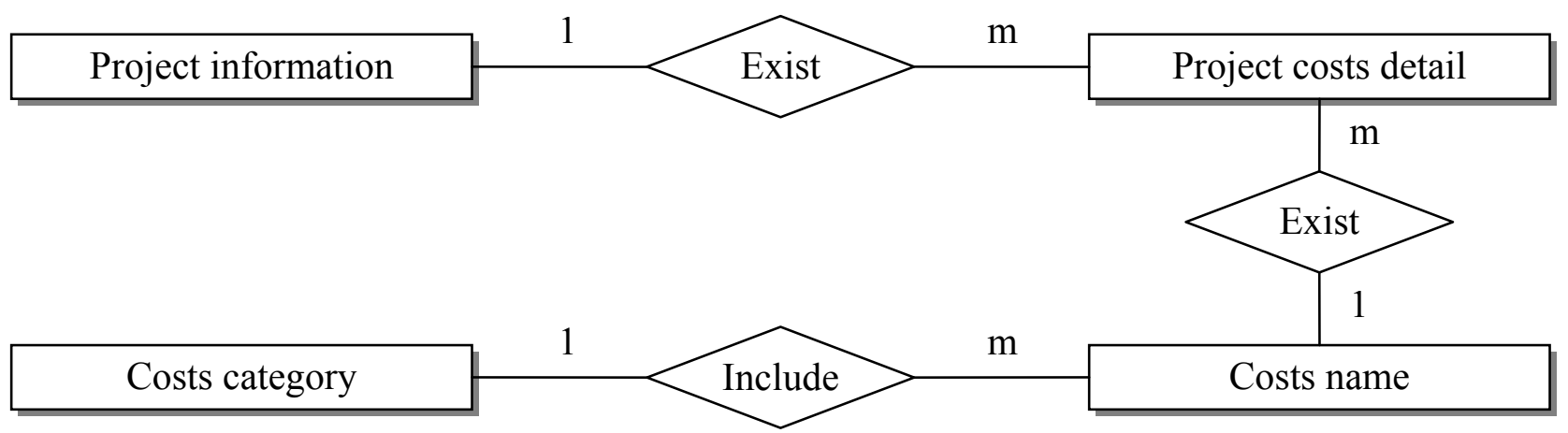

Fig. 1. Entity relationship diagram on construction project cost system

\section{Data Types on DB2}

The system is based DB2 database management system, the advantages as follows: openness and portability, from the network protocol support to programming interface, database stored procedures and triggers, distributed operation between servers in development of the application, are based on the latest international standards; processing performance, IBM invented the relational database and optimization techniques in a variety of performance tests enjoys a leading position; parallelism, DB2 database management expanded to the parallel and multi-node environment; distributed database, processing and Connectivity built on the core functions of DB2, without the use of other products annex; replication capabilities, DB2 provides the industry's best and most efficient open copy program to provide automatic conflict detection and resolution; e-commerce support, providing JDBC interface, available Java language to write stored procedures and user-defined functions; software quality and reliability, DB2 is a database of all manufacturers in the highest reliability database lowest failure rate. When storing data in the table, the space occupied different types data and the storage way are not the same [3, 4]. DB2 is divided into two kinds of data types, built-in data types and user-defined data types. Built-in data types include "String, Numeric, Datetime and XML" and other four categories, as shown in Fig. 2.

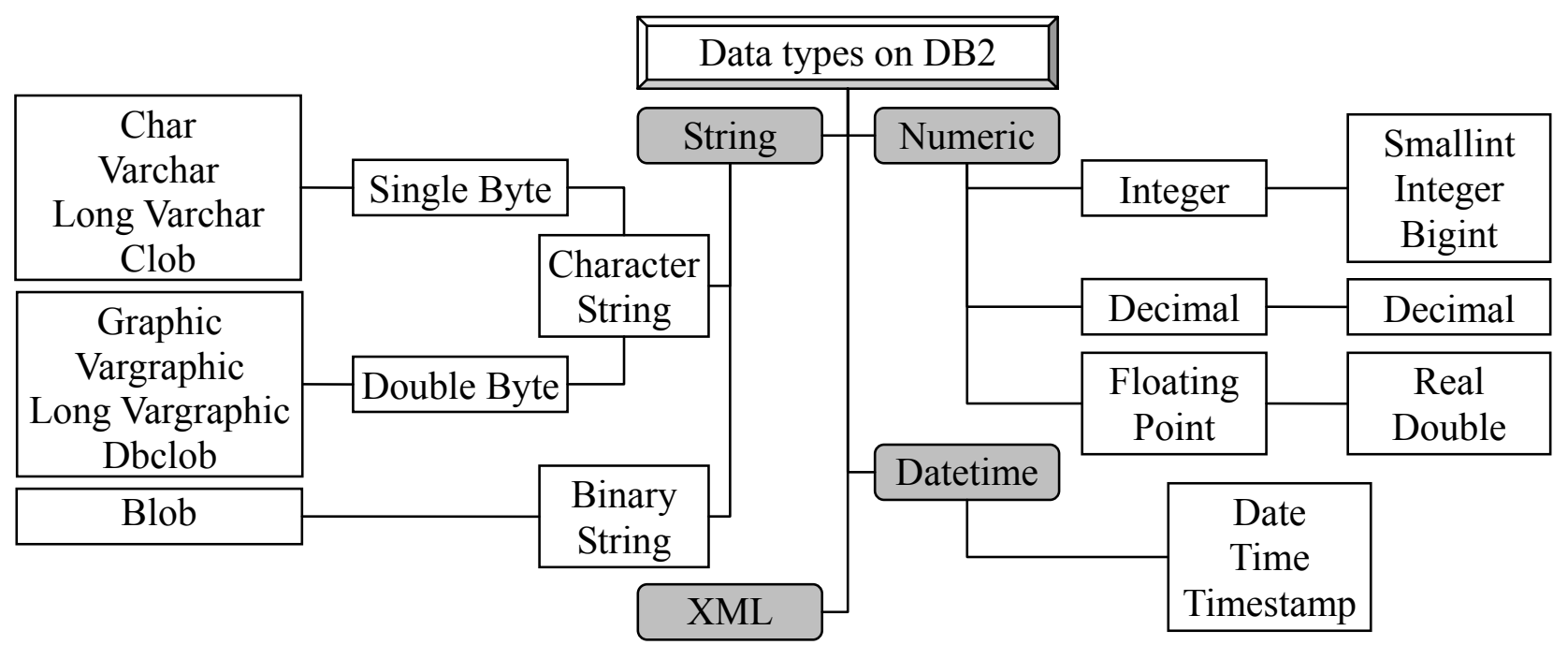

Fig. 2. Data types on DB2 database management system 


\section{Logical Structure Design}

Unified modeling language (UML) is a comprehensive modeling techniques on the basis of a Booch, OMT and OOSE method, using case diagrams, class diagrams, interaction diagrams and activity diagrams model describe the whole picture of complex systems and their associated components contact. Among them, the class diagram describes the static structure of the system for a variety of classes and objects. In a relational database field, type and correspond to the table. This paper used the strategy that the class and its objects in the UML class diagram are mapped to tables in a relational database. Mapping results is shown in Fig. 3.

\begin{tabular}{|c|}
\hline Project information table \\
\hline Primary key identity (IDzgjz, Integer, 4) \\
\hline Project encoding (XBXXxmbm, Char, 8) \\
\hline Project name (XBXXxmmc, Varchar, 100) \\
\hline Phoneticize simple encoding (XMXXpyjm, Varchar, 10) \\
\hline Project address (XBXXxxdz, Varchar, 100) \\
\hline Contact telephone (XBXXlxdh, Varchar, 50) \\
\hline Contact electronic mail (XBXXlxyx, Varchar, 100) \\
\hline Manager name (XBXXfyzxm, Varchar, 30) \\
\hline Manager telephone (XBXXfzrdh, Varchar, 50) \\
\hline Plan commencement date (XBXXkgrq, Date, 4 ) \\
\hline Plan complete date (XBXXwgrq, Date, 4 ) \\
\hline Project brief introduction (XBXXxmjj, Long Varchar, 5000)) \\
\hline One-to-Many (XBXXxmbm) \\
\hline Project costs detail table \\
\hline Primary key identity (IDzgjz, Integer, 4) \\
\hline Project encoding (XBXXxmbm, Char, 8) \\
\hline Costs encoding (FYMCfybm, Char, 4) \\
\hline Costs amount (XMZJfyje, Decimal, 10,2) \\
\hline Budget personnel encoding (XMZJysrybm, Varchar, 50) \\
\hline Budget date time (XMZJysrqsj, Timestamp, 7) \\
\hline Budget basis (XMZJysyj, Long Varchar, 1000) \\
\hline One-to-Many (FYMCfybm) \\
\hline Costs name dictionary \\
\hline Primary key identity (IDzgjz, Integer, 4) \\
\hline Costs category encoding (FYLBlbbm, Char, 2) \\
\hline Costs category name (FYLBlbmc, Varchar, 50) \\
\hline Costs encoding (FYMCfybm, Char, 4) \\
\hline Costs encoding name (FYMCfymc, Varchar, 50) \\
\hline Phoneticize simple encoding (FYMCpyjm, Varchar, 10) \\
\hline Costs name explain (FYMCfysm, Long Varchar, 1000) \\
\hline One-to-Many (FYLBlbbm) \\
\hline Costs category dictionary \\
\hline Primary key identity (IDzgjz, Integer, 4) \\
\hline Costs category encoding (FYLBlbbm, Char, 2) \\
\hline Costs category name (FYLBlbmc, Varchar, 50) \\
\hline Phoneticize simple encoding (FYLBpyjm, Varchar, 10) \\
\hline Costs category explain (FYLBlbsm, Long Varchar, 1000) \\
\hline
\end{tabular}

Fig. 3. Relation class diagram on construction project cost system 


\section{Storage Structure Design}

DB2 storage structure is very important for a reasonable database physical design. In DB2 systems, data storage is defined as four layers, as shown in Fig. 4. In which, "Partition Group" is a partition exists in the multi-level database. A table space contains one or more containers, but at least one. The container can be an operating system directory, file, or predefined size unformatted raw device (such as a hard disk, Windows disk partition, AIX logical volume, etc.). Tables, indexes, and other database objects created by the user stored in the table space. Table direct application-oriented, while the table again, and physical storage space corresponding to the bottom of the table space can have more than one container, the container is stored on the ground floor [5, 6]. So according by table space database achieved the unity of physical storage and logical storage.

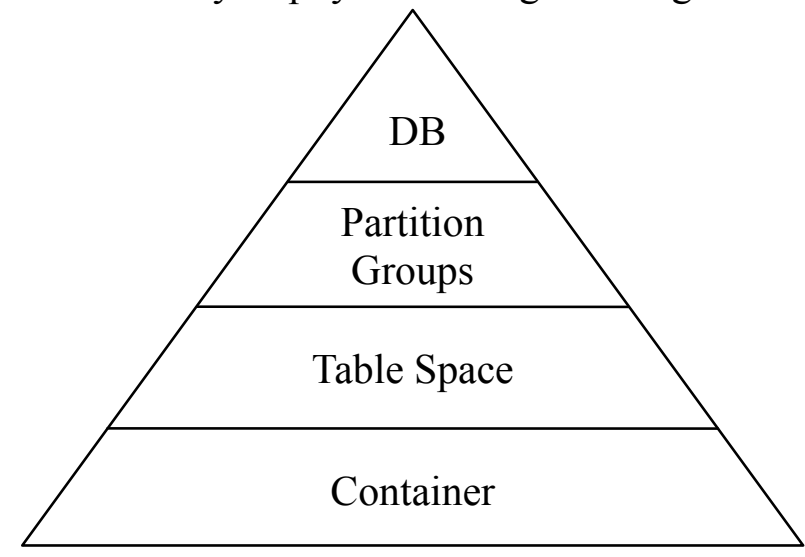

Fig. 4. Storage hierarchical structure of DB2

\section{Index Design}

Creating an index can improve query speed, ensure data uniqueness. DB2 includes four index [7,8]: First, non-unique index, the index most of the non-unique index, which is related to the distribution and data, general data are reproducible characteristics, can not be defined as a unique index; two is the only index used to ensure uniqueness, performance than non-unique index, this index is related to the degree and dense data. Dense degree unique index is equal to the total number of data reciprocal; the third is completely index is relative to the general index, the use of "CREATE INDEX ... INCLUDE" statement when creating an index, you can include additional columns of data, with the key stored together, but it is not part of the key, so do not be sorted. Complete index can reduce the access to the data pages; fourth, the cluster index, the logical order of the key determines the physical order of the corresponding rows in the table. That is when a clustered index on a clustered index, and create a clustered index, you need to reorder the existing table data, that is, after deleting the original data table, and then sort the results back into the physical order, so the clustered index after the establishment, the establishment of the clustered index data columns have all been arranged sequentially. A table can contain only one clustered index, but the index can contain multiple columns. "B-Tree" tree index, the leaf level of the clustered index is the data page.

The achievement of DB2 index is a B+ tree, can achieve fast query, and avoid full table scans, to reduce the IO operation. The index is an abstract data table, by extracting limited data on the distribution of the data is calculated in order to complete the fast retrieval of data. Create an index should follow the following principles: The index includes the "WHERE" clause in all the columns, the index is formed by using a shield, rejected the result set failed row; for the SQL statement "RODER BY" clause references to column, create an index appropriate to avoid the sort; taking into account the cost of management, should be avoided in the index using more than five columns; for multi-column indexes, most referenced in the query definition on the front row; cardinality large columns to create an index for; do not include those that are commonly modified or regular insert, 
delete in the index. The only exception is the primary key and foreign key; can not use "null" to index any column that contains "null" values are not to be included in the index, any use of "is null" or the "WHERE" clause "is not null" statement optimizer is not using an index; used "include" keyword to create the index.

This system is mainly to create two types of indexes: one, the only index used to ensure that data is unique; the two is non-unique index to improve query speed. As shown in Table 1.

Table 1. Index design on construction project cost system

\begin{tabular}{|c|c|c|c|}
\hline Type & Index name & Table name & Include columns \\
\hline \multirow{4}{*}{$\begin{array}{l}\text { Unique } \\
\text { index }\end{array}$} & IDX_ProjectInformation_1 & Project information table & XBXXxmbm \\
\hline & IDX_ProjectCostsDetail_1 & Project costs detail table & XBXXxmbm , FYMCfybm \\
\hline & IDX_CostsName_1 & Costs name dictionary & FYMCfybm \\
\hline & IDX_CostsCategory_1 & Costs category dictionary & FYLBlbbm \\
\hline \multirow{4}{*}{$\begin{array}{l}\text { Non-unique } \\
\text { index }\end{array}$} & IDX_ProjectInformation_2 & Project information table & $\begin{array}{l}\text { XBXXxmbm, XBXXxmmc, } \\
\text { XBXXfyzxm, XBXXkgrq }\end{array}$ \\
\hline & IDX_ProjectCostsDetail_2 & Project costs detail table & $\begin{array}{l}\text { XBXXxmbm, FYMCfybm, } \\
\text { XMZJfyje, XMZJysrybm }\end{array}$ \\
\hline & IDX_CostsName_2 & Costs name dictionary & $\begin{array}{l}\text { FYLBlbbm, FYLBlbmc, } \\
\text { FYMCfybm, FYMCfymc }\end{array}$ \\
\hline & IDX_CostsCategory_2 & Costs category dictionary & FYLBlbbm, FYLBlbmc \\
\hline
\end{tabular}

\section{Conclusion}

With the development of large-scale socialized production, the exploration and open of construction market, large amounts of data in project cost management services urgently need for rapid and scientifically accurate and timely decisions. Widely used in computers, the unprecedented development of network technology, the integration and high penetration of multimedia in the field of construction project provides a solid platform for the modernization of the means of change and project cost management. Database design is the basis work for system development, this paper fully combined the characteristics of DB2 database management system, designed by step of database design for the whole process, provides a viable solution for system development, to accelerate the construction project cost system development has an important role.

\section{References}

[1] Z. J. He, H. D. Cao, Y. Y. Zi, et al, "Developments and Thoughts on Operational Reliability Assessment of Mechanical Equipment," Journal of Mechanical Engineering, vol. 50, no. 2, pp. 171-186, 2014.

[2] M. J. Chen, "Maintenance and management of mechanical equipment from the perspective of economic operation," China Management Informationization, vol. 16, no. 10, pp. 107-108, 2013.

[3] L. Z. Miao, "Management measures for safety operation of mechanical equipment," Journal of Henan Science and Technology, vol. 48, no. 16, pp. 77-78, 2013.

[4] Baidu Encyclopedia, "Conceptual Structure Design," http://baike.baidu.com/view/1638403.htm, 2016-1-10.

[5] L. Zhang, L. Ma, "Database Design," Journal of Anyang Institute of Technology, vol. 6, no. 4, pp. 76-79, 2007. 
[6] Z. Y. Duan, "Database Design Method," Journal of Nanchang College of Education, vol. 20, no. 4, pp. 84-86, 2005.

[7] Y. Zhang, "Eliminating Process of Formalization in Logical Database Design," Computer Systems \& Applications, vol. 22, no. 6, pp. 179-181, 2013.

[8] doc88, "Logical structure design," http://www.doc88.com/p-117617790552.html, 2015-2-10.

[9] H. M. Chen, "Logical structure design of database," Fujian Computer, vol. 28, no. 10, pp. 214-215, 2012.

[10] J. S. Yang, C. Zhang, "Effect of index on SQL execution cost," Information communication, vol. 27, no. 1, pp. 136-137, 2013. 